\title{
ARTICLE Apatinib, a selective VEGFR2 inhibitor, improves the delivery of chemotherapeutic agents to tumors by normalizing tumor vessels in LoVo colon cancer xenograft mice
}

\author{
Kai Zhou ${ }^{1}$, Jing-wei Zhang ${ }^{1}$, Qi-zhi Wang ${ }^{1}$, Wen-yue Liu ${ }^{1}$, Jia-li Liu ${ }^{1}$, Lan Yao ${ }^{1}$, Ming-min Cai ${ }^{1}$, Sui-ying Ni ${ }^{1}$, Qing-yun Cai ${ }^{1}$, \\ Guang-ji Wang ${ }^{1}$ and Fang Zhou ${ }^{1}$
}

Tumor vascular normalization has been proposed as a therapeutic strategy for malignant neoplasms, which can also interpret the synergistic effect of anti-angiogenesis agents combined with chemotherapy. Apatinib (Apa), a highly selective VEGFR2 inhibitor, attracts much attentions due to its encouraging anticancer activity, especially in the clinical trials of combined treatment. In this study, we investigated whether Apa could promote vascular normalization in tumor in a certain time window. Mice bearing LoVo colon cancer xenograft were orally administrated Apa (150 $\mathrm{mg} \mathrm{kg}^{-1}$ per day) for $5,7,10$, or 12 days. Apa significantly inhibited tumor growth and decreased the microvessel density. Using multi-photon microscopy and electron microscopy, we found that Apa improved tumor vessel morphology by pruning distorted vessel branches and decreased the gap between endothelial cells after a 7-day treatment. Furthermore, Apa decreased vessel leakage and increased pericyte coverage on vascular endothelial cells, suggesting that tumor vessels were more mature and integrated. The intratumoral distribution of adriamycin (ADR) in Apa group was improved from day 7 to 10 without change in plasma drug concentration. Tumor blood perfusion was also increased in this window, and the expression of hypoxia induced factor 1a was downregulated, suggesting the effect of Apa on alleviating tumor hypoxic micro-environment. In conclusion, Apa may improve the effective perfusion of tumor vessels and increase the intratumoral distribution of ADR in a certain time window via normalizing tumor vessels. This normalization window ( 7 to 10 days of treatment) may contribute to develop a regimen of combined medication in clinic use of Apa.

Keywords: apatinib; anti-angiogenesis; tumor vessel; normalization window; combined therapy; adriamycin; colon cancer.

Acta Pharmacologica Sinica (2019) 40:556-562; https://doi.org/10.1038/s41401-018-0058-y

\section{INTRODUCTION}

Overactivated angiogenesis is one of the hallmarks of cancer. As cancer cells require excessive energy and nutrition supply during rapid proliferation, the activated vasculature continually sprouts new immature vessels to acquire a blood supply [1]. Antiangiogenesis strategies can effectively suppress tumor growth by reducing the energy supply of cancer cells [2] Currently, increasing numbers of anti-angiogenesis drugs are being developed for cancer therapy [3, 4]. Moreover, accumulating data indicate that some anti-angiogenic drugs can also potentiate the effects of radiation and chemotherapy [5-7] To explain this synergistic phenomenon, J. Kain put forward the hypothesis of the "Tumor Vascular Normalization Window" [8]. In this hypothesis, anti-angiogenic therapy can induce a temporary reversion of tumor vessels towards a normalized phenotype that includes normalized vessel structure and function. These improvements are generally accompanied by enhanced delivery of chemotherapeutic drugs into the tumor. Notably, the normalization effect is transient and reversible [9] and only an appropriate dosing time can cause tumor vessel normalization. Combination treatments applied at inappropriate times have been reported to be unable to enhance the therapeutic effect and can even decrease the delivery of chemotherapeutic drugs into tumors [10-12]. Therefore, identifying the normalization window for anti-angiogenesis agents is crucial for the use of combinations in the clinic.

Apatinib (Apa) is an orally dosed tyrosine kinase inhibitor of vascular endothelial growth factor 2 (VEGFR2) with a binding affinity that is 10 times greater than that of sorafenib [13]. As the first small molecular anti-angiogenesis agent that has been found to be effective for advanced gastric cancer patients who have no chemotherapy indications [14], Apa was approved by the China Food and Drug Administration for the third-line treatment of gastric cancer in China. Moreover, due to promising anti-tumor effects in multiple solid cancers, such as lung cancer [15], colon cancer [16], and nasopharyngeal carcinoma [17], Apa has been involved in some second-line treatments in clinical trials. Currently, there are many successful findings about the combination of Apa with other conventional chemotherapeutic drugs in animal experiments [18, 19] and clinical trials [20, 21], but direct evidence proving that Apa has a vascular normalization effect from which combination therapies could benefit is still lacking.

${ }^{1}$ Key laboratory of Drug Metabolism and Pharmacokinetics, State Key Laboratory of Natural Medicines, China Pharmaceutical University, Nanjing 210009, China Correspondence: Guang-ji Wang (guangjiwang@hotmail.com) or Fang Zhou (zf1113@163.com)

These authors contributed equally: Kai Zhou, Jing-wei Zhang.

Received: 12 January 2018 Accepted: 31 May 2018

Published online: 5 July 2018 
(a)

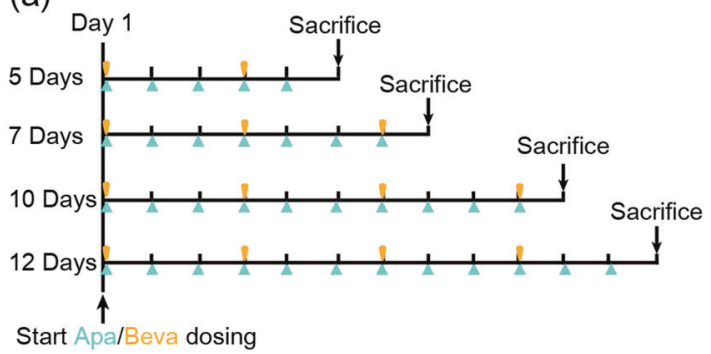

(c)

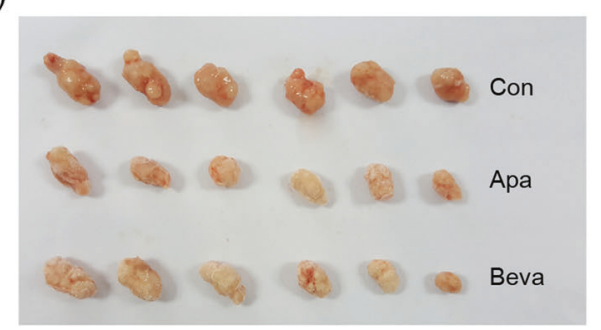

(b)

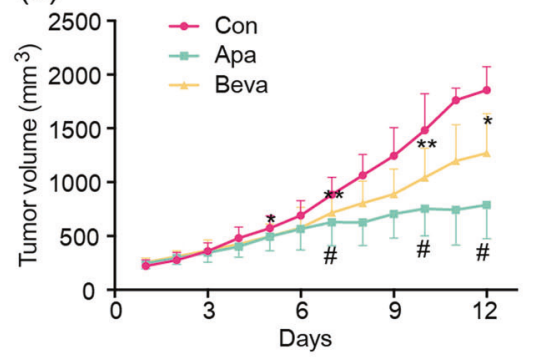

(d)

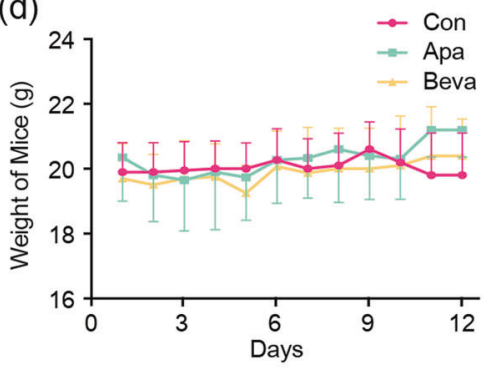

Fig. 1 Anti-tumor effect of Apa in LoVo colon cancer xenograft mice. a Schematic plans for the administration of anti-angiogenesis agents and animal experiments. $\mathbf{b}$ Tumor volumes after the Apa and Beva treatments. ${ }^{*} P<0.05,{ }^{* * *} P<0.01$, and ${ }^{\#} P<0.01$ vs. the Con group. $\mathbf{c}$ Images of LoVo tumors resected from the mice after 12 days of treatment. $\mathbf{d}$ Mouse weights during the experiments

We hypothesize that if Apa can normalize tumor vessels within a definite time window, it would be possible to precisely handle the schedule of combination use and amplify the potential effect of Apa in cancer treatment. The aims of this study were to explore the effect of Apa on normalizing tumor vessels in colon cancer xenografts and to identify the normalization window that can increase the accumulation of chemotherapeutic agents in the tumor.

\section{MATERIALS AND METHODS}

Animal experiments

Balb/c nude mice of 6-8 weeks of age weighing $18-22 \mathrm{~g}$ and bearing LoVo xenografts were obtained from SLAC Laboratory Animal Co., Ltd. (Shanghai, China). All mice were maintained in a special pathogen-free environment and were used with the approval of the Animal Ethics Committee of China Pharmaceutical University.

The lengths (major tumor axis) and widths (minor tumor axis) of the tumors were measured with a caliper every day, and the tumor volumes were calculated with the following formula: $V=$ $0.5 \times$ length $\times(\text { width })^{2}$. The experiments were initiated when the tumor volume reached $200 \mathrm{~mm}^{3}$. The mice were randomly divided into three groups: a Control (Con) group, an Apatinib (Apa) group, and a Bevacizumab (Beva) group ( $n=6$ in every group at every time point). In the Con group, the mice were orally administered saline each day. In the Apa group, the mice were orally administered Apa solution $(150 \mathrm{mg} / \mathrm{kg}$, dispersed in $0.5 \%$ CMC$\mathrm{Na}$ solution) each day. In the Beva group, the mice were intravenously administered with Beva $(5 \mathrm{mg} / \mathrm{kg})$ every 3 days. To investigate the effects of the anti-angiogenesis agents at different time points, the mice were maintained on the dosing schedules for $5,7,10$, or 12 days. Killing of the animals and sampling were conducted on days $6,8,11$, and 13 (Fig. 1a).

Intravital multi-photon laser scanning microscopy

After the injection of $10 \mathrm{mg} / \mathrm{kg}$ fluorescein isothiocyanate (FITC)labeled dextran (Sigma-Aldrich, St Louis, USA), the mice were immediately anesthetized with pentobarbital. The skin around the tumor was carefully removed and the tumor was fixed onto selfmade equipment to prevent the tremble caused by the heartbeat during observation. A $\times 20$ lens was used for observation. Z-stacks were acquired to a depth of 200-300 $\mu \mathrm{m}$ beneath the surface of the tumor. Three-dimensional reconstruction of the tumor vessels was accomplished with ZEN software (Zeiss, Germany).

\section{Scanning electron microscope imaging}

The stripped tumors were immediately cut into small pieces $(6 \mathrm{~mm}$ in length and $4 \mathrm{~mm}$ in width) and fixed with $2.5 \%$ glutaraldehyde solution at $4{ }^{\circ} \mathrm{C}$. The fixed tumors were dehydrated in a graded acetone series, dried at $40^{\circ} \mathrm{C}$ for over $48 \mathrm{~h}$, stuck to the object stage with the sections upwards, and coated with gold. Next, the tissues were photographed at $2.5 \mathrm{~K}$-fold magnification using a Hitachi UHR FE-SEM (SU8000, Hitachi, Japan).

\section{Immunofluorescence}

The tumors were collected and fixed in $4 \%$ paraformaldehyde (PFA) at $4{ }^{\circ} \mathrm{C}$ for $8 \mathrm{~h}$ and dehydrated by soaking the tissues blocks in a $20 \%$ sucrose solution for $24 \mathrm{~h}$. The tissues were embedded in optimum cutting temperature compound (Sakura, USA) and sectioned at $10 \mu \mathrm{m}$. To detect the vascular endothelial cells and calculate the tumor microvascular density (MVD), the sections were incubated with R-phycoerythrin (PE)-labeled anti-CD31 (1:50, $\mathrm{BD}, \mathrm{USA}$ ) overnight at $4^{\circ} \mathrm{C}$. For the pericyte coverage experiment, the sections were incubated with PE-labeled anti-CD31 (1:50, BD) and anti-a-SMA (1:100, Abcam, UK) overnight and then incubated with FITC-conjugated secondary antibody (1:200, Bioworld, USA) for $1 \mathrm{~h}$ at $37^{\circ} \mathrm{C}$. To detect the leakage of the tumor vessels, FITClabeled dextran was injected and allowed to circulate for $15 \mathrm{~min}$ before the mice were killed. Vascular endothelial cells were labeled with PE-CD31 antibody and the leaked dextran was directly observed with a confocal microscope (LSM 700, Zeiss). For the morphometric and quantitative analyses, five random optical fields per tumor section were collected and the image data were analyzed using ImageJ $(\mathrm{NIH})$.

\section{Immunohistochemistry}

The tumors were fixed in 4\% PFA after dissection from the animal and prepared into paraffin sections. Next, the sections were deparaffinized and hydrated in ethyl alcohol. The antigen retrieval process was performed in $10 \mathrm{mM}$ sodium citrate buffer for $20 \mathrm{~min}$ 
(a)

(a) 5 Days

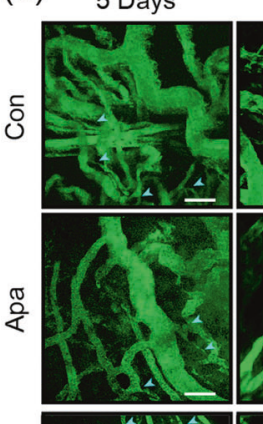

7 Days

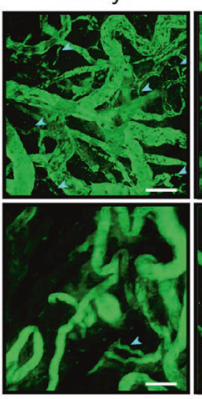

10 Days

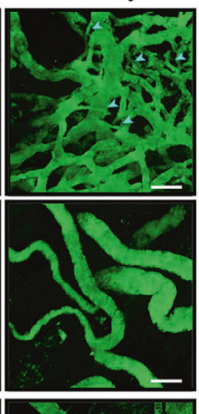

12 Days

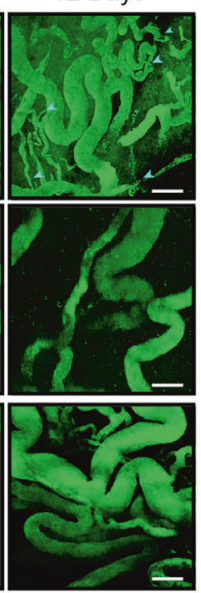

(b)

(b)

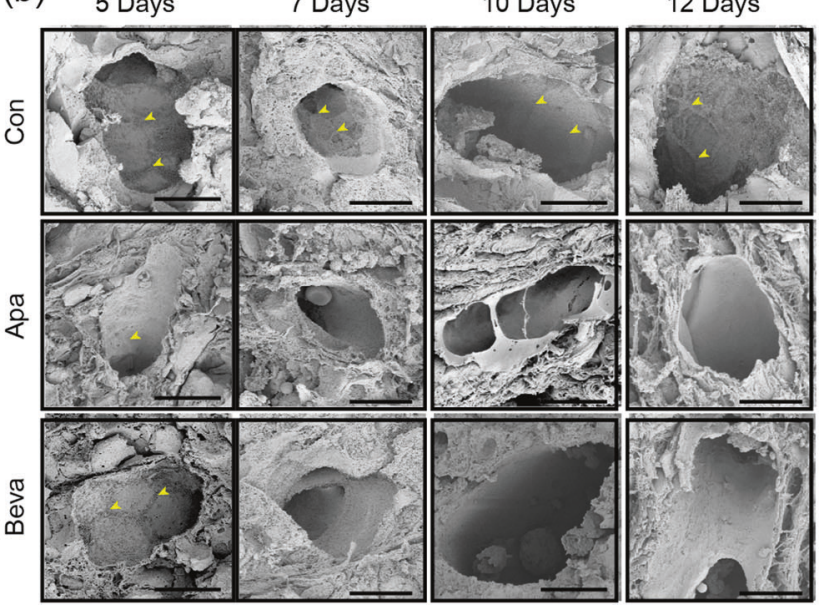

Fig. 2 Morphologies and structures of the tumor vessels. a Morphologies of the tumor vessels as monitored by multi-photon laser scanning microscopy (Zeiss). Disordered vessels are indicated by blue arrows (scale bar, $100 \mu \mathrm{m}$ ). b SEM photographs of the lumina of tumor vessels. The gaps between the endothelial cells are indicated by yellow arrows (scale bar, $10 \mu \mathrm{m}$ )

using a microwave oven. After blocking, the sections were incubated overnight at $4{ }^{\circ} \mathrm{C}$ with primary anti-HIF-1a antibodies (1:200, Abcam). The two-step technique (SuperPictureTM3rd Gen IHC Detection Kit, Invitrogen, USA) was used for visualization and DAB (Vector Laboratories, USA) served as a chromogen. Images were collected with an inverted microscope (Leica, Germany).

Drug concentration measurement

To avoid direct drug-drug interactions, the effects of the antiangiogenesis agents on the intra-tumor distributions of adriamycin (ADR) were detected on days 6, 8, 11 and 13 . At $1 \mathrm{~h}$ after the intravenous administration of ADR $(15 \mathrm{mg} / \mathrm{kg})$, the mouse plasma and tumors were collected. The concentration of ADR was measured with a liquid chromatography (LC) (LC20A, Shimadzu, Japan) tandem mass spectrometry (MS/MS) (Triple Quad 5500, SCIEX, USA) system. The detailed conditions were as follows: $544.2 / 361.0 \mathrm{~m} / \mathrm{z}$ of ADR and 270.0/181.0 m/z of diphenhydramine (internal standard). The separation process was performed on a Luna $\mathrm{C}_{8}$ column $(5 \mu \mathrm{m}, 2.0 \times 150 \mathrm{~mm}$, Phenomenex, USA). The mobile phases consisted of water containing $0.1 \%$ formic acid and methanol.

Laser speckle flow imaging of tumor blood flow

After the mice were anesthetized with pentobarbital, the skin around the tumor was cut to exclude interference caused by skin vessels. The mice were placed on a pad with the tumor upward and examined with a laser speckle contrast analysis imager (moorFLPI, UK). At least 20 pictures were collected at an interval of $2 \mathrm{~s}$ for each mouse. The blood flux data were analyzed with moorFLPI viewer software.

\section{Statistical analysis}

All statistical analyses were conducted using GraphPad Prism 7 (GraphPad Software, Inc., San Diego, CA, USA). Student's t-tests, or one-way analysis of variances were used to compare the mean values and the results are presented as the mean \pm SD. $P$-values $<$ 0.05 were considered significant.

\section{RESULTS}

The anti-tumor effect of Apa on LoVo xenografts

To investigate the normalization window of Apa on LoVo xenografts, the tumors were harvested on days 6, 8, 11, and 13 . As presented in Fig. 1b, c, Apa significantly inhibited tumor growth from days 7 to day 12 compared with the Con group. On day 5 , the mean tumor volume in the Apa group begin to exhibit a reduction compared with the Con group. The tumor volume curve of the Apa group was even lower than that of the Beva group (i.e., the positive control). The mice were weighed every day to evaluate the potential toxicities of the anti-angiogenesis agents. As illustrated in Fig. 1d, neither the Apa nor Beva group exhibited any adverse changes in weight, which indicated that there was no significant systematic toxicity.

Apa improved the morphology and microstructure of the tumor vessels

FITC-dextran was used as a tracer to label the perfused vessels. After laser scanning and $3 \mathrm{D}$ reconstruction, the vascular structure and morphology were visualized. As presented in Fig. 2a, disordered and crossed small vessel branches were observed in the Con group at different time points. In the Apa group, these tiny branches had been pruned and fixed after Apa treatment for 7 days and had nearly vanished after 12 days of treatment. Beva also elicited a vessel improving effect in this model.

Next, the effects of Apa on the endothelial cells of the tumor vessels were investigated by scanning electron microscope (SEM) imaging. Activated endothelial cells with clear edges and rough surfaces were observed in the Con group (Fig. 2b). In the Apa group, the lumina of the vessels were smooth, and the edges between endothelial cells disappeared after 7 days of treatment. Treatment with the VEGF blocker Beva (positive control) elicited a normalizing effect that was similar to that observed in the Apa group.

Apa reduced the tumor MVD

Frozen tumor sections were stained with PE-labeled CD31 to label the tumor vascular endothelial cells. As illustrated in Fig. 3a, both the Apa and Beva treatments remarkably decreased the CD31positive cells in the tumors compared with the Con group. Quantitative analysis also revealed that the MVDs were significantly decreased in the Apa and Beva groups at every time point (Fig. 3b).

Apa decreased vessel leakage and increased pericyte coverage To further study the integrity and maturity of the tumor microvessels, the leakage and pericyte coverage were investigated at different time points. Seven-day treatment with Apa resulted in less FITC-dextran leakage from the tumor vessels compared with that observed in the Con group (Fig. 4a, b), which indicated improved vessel integrity and function. 
(a)

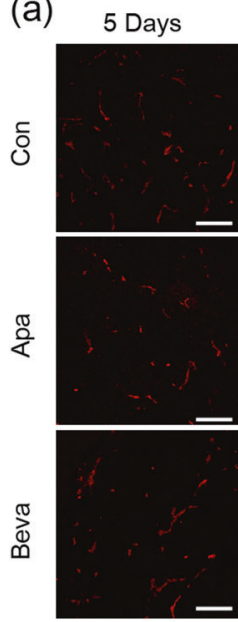

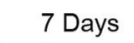

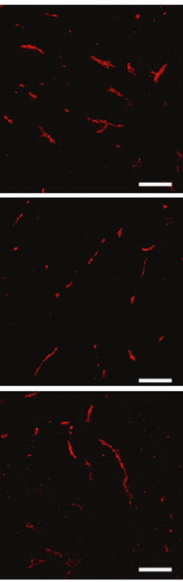
10 Days

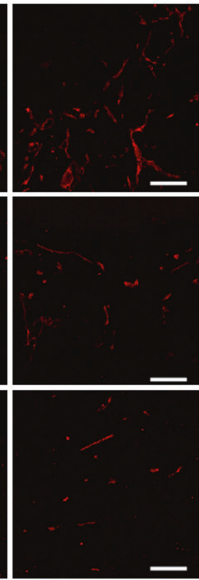

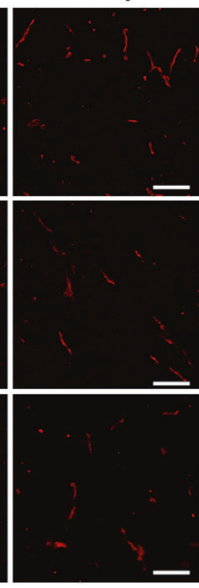

(b)
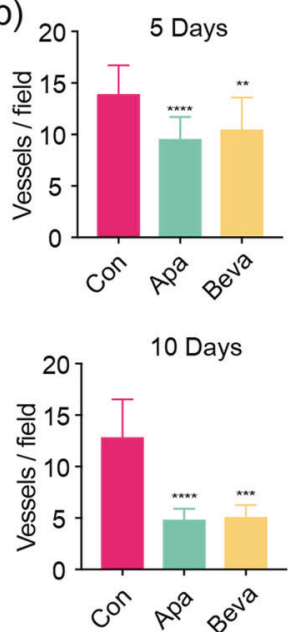

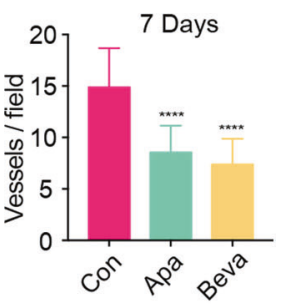

12 Days

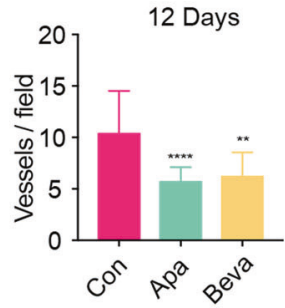

Fig. 3 Immunofluorescence of the MVDs in the tumors. a Tumor vascular endothelial staining with PE-CD31 (scale bar, $100 \mu m$ ). $\mathbf{b}$ Statistical

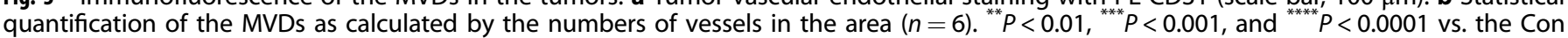
group

The neovasculature in tumors usually exhibits a state of immaturity as indicated by the lack of pericyte coverage around the vascular endothelial cells. a-SMA antibody was used for pericyte immunostaining. When a-SMA was localized around the CD31-positive cells, the vessel was regarded as an a-SMA-positive vessel. As illustrated in Fig. 4c, d, Apa significantly improved the ratio of a-SMA-positive to -negative vessels after treatment for 7 days, which demonstrated that the vessels were more mature and normalized. In contrast, the pericyte coverage in the Con group remained at a level of $20 \%$ at all time points.

Apa increased the distribution of ADR in the tumor tissues The concentrations of ADR in the tumors were detected by LC-MS/ MS to investigate the influence of Apa on drug distribution. With tumor growth, the intratumoral concentration of ADR in the Con group gradually decreased from day 5 to day 12 (Fig. 5a). However, the pattern was different in the Apa group. The intratumoral concentrations of ADR after the 7-day and 10-day treatments were higher than those after the 5-day and 12-day treatments. Moreover, following the 7-day and 10-day treatments, the intratumoral concentrations of ADR in the Apa group were significantly higher than those of the corresponding Con group treatments. To investigate whether the altered intratumoral concentrations were related to the plasma concentrations, the concentrations of ADR in the plasma were also measured. There were no significant differences between the groups (Fig. 5b), which indicated that some other mechanism was responsible for the increased ADR concentrations in the tumors following Apa treatment and that there were no significant drug-drug interactions between Apa and ADR at the plasma level. These data revealed that ADR could be better distributed into the tumor only in a specific time range and further suggested a possible tumor vessel normalization window for Apa of 7 to 10 days of treatment.

Apa improved tumor blood perfusion and downregulated the expression of HIF-1a in the vascular normalization window As vessel structure and drug delivery were improved within the normalization window, the tumor micro-environment in this normalization window was next investigated from the perspective of blood perfusion and the degree of hypoxia in the tumor. The dynamic blood perfusion was directly monitored and recorded with a laser speckle contrast analysis imager. As illustrated in Fig. 6 $a, b$, in the Con group, tumor blood perfusion gradually decreased with tumor growth regardless of whether perfusion was examined inside or outside of the normalization window. Blood perfusion was significantly improved in the Apa group within the normalization window, whereas it dropped dramatically to the level observed in the Con group with the vanishing of the normalization window.

Considering that the degree of hypoxia in the tumor microenvironment might reflect changes in blood perfusion, we investigated the expression of HIF-1a, which is a hypoxia marker that is closely related to angiogenesis, to validate the alterations of the tumor micro-environment. As presented in Fig. $6 c$, d, the expression of HIF-1a was significantly downregulated within the normalization window in the Apa group, whereas it recovered to a level close to that in the Con group outside of the normalization time. These data confirmed the improvement of the hypoxic micro-environment of the tumor within the normalization window after Apa treatment.

\section{DISCUSSION}

Conventional chemotherapy usually inhibits tumor growth by straightforwardly damaging cancer cells. However, conventional chemotherapy may cause severe side effects due to nonselective cytotoxicity. Moreover, distorted tumor vessel nets may reduce drug delivery into the tumor and thus limit the therapeutic effects. Anti-angiogenesis agents inhibit tumor vascular density to reduce the nutrition supply to the tumor, but the excessive inhibition of tumor vessel proliferation may result in hypoxia and even the migration of malignant cancer cells. This process is thought to be related to the poor prognoses of cancer patients after long-term anti-angiogenesis treatment [22]. Thus, a better strategy was proposed, i.e., the utilization of the normalization effect of antiangiogenesis agents on tumor vessels to improve the delivery of chemotherapeutic drugs into the tumor. Several marketed antiangiogenesis agents exhibit synergistic therapeutic effect when combined with chemotherapy or radiotherapy [23-25]. However, whether Apa can elicit tumor vascular normalization to enhance the effect of chemotherapy remains unclear. Treatment with Apa combined with chemotherapeutic drugs has presented promising therapeutic effects [18-21]. In the relevant published papers, the synergistic effects of Apa with chemotherapeutic agents have been attributed to the inhibition of multidrug resistance-related efflux transporters. Our present study demonstrated that Apa promoted tumor vascular normalization within a 
(a)
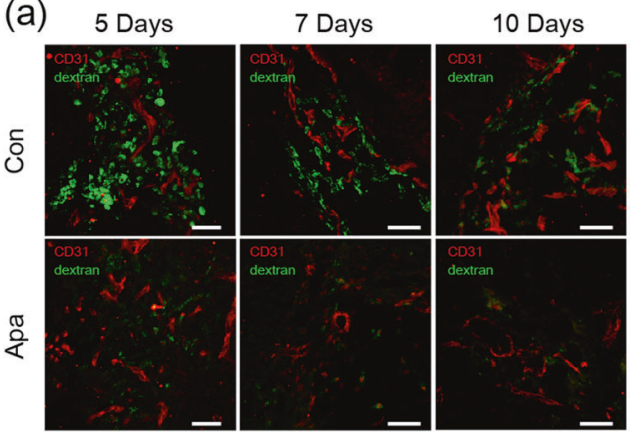

(c)

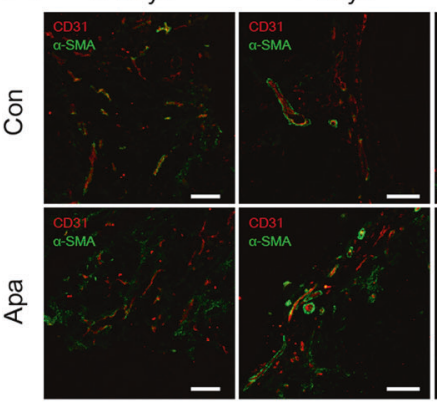

10 Days

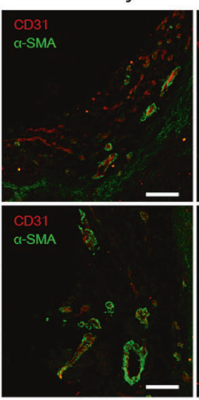

12 Days

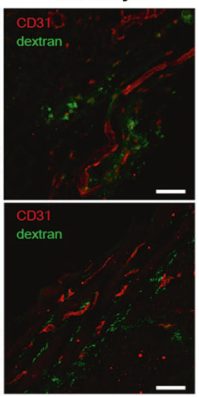

12 Days

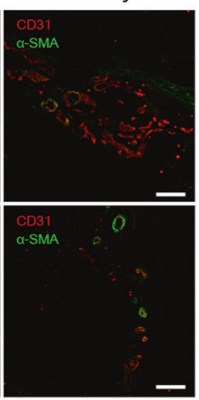

(b)

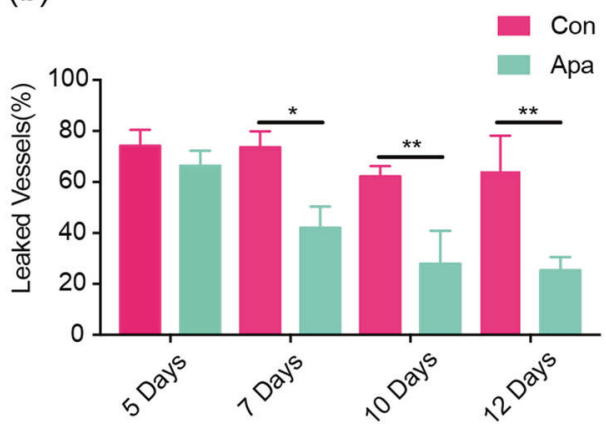

(d)

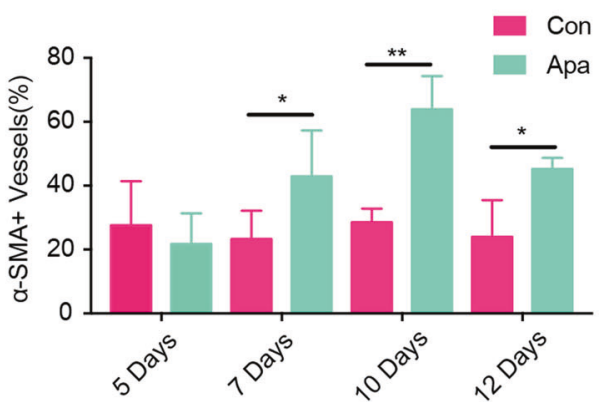

Fig. 4 Immunofluorescence for dextran leakage and pericyte coverage. Effect of Apa on FITC-dextran leakage. The tumor vessels were marked with CD31 (red). a Leaked dextran was directly observed by laser confocal microscopy (scale bar, $50 \mu \mathrm{m}$ ). b Statistical quantification of the percentages of leaking vessels $(n=3))^{*} P<0.05$ and ${ }^{* * *} P<0.01$ vs. the Con group. c Double staining for CD31 (red) and $\alpha-S M A$ (green) revealing pericyte coverage in the Con and Apa groups (scale bar, $100 \mu \mathrm{m}$ ). $\mathbf{d}$ Statistical quantification of the pericyte coverage based on calculations of the $\alpha$-SMA + fractions $(n=3) .{ }^{*} P<0.05$ and ${ }^{* *} P<0.01$ vs. the Con group

(a)

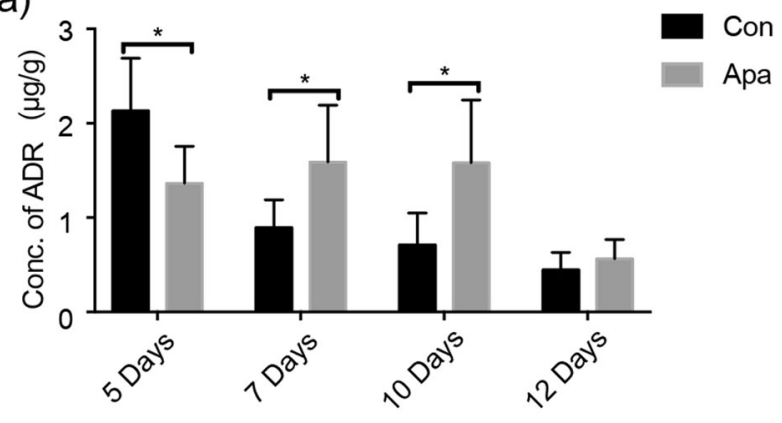

(b)

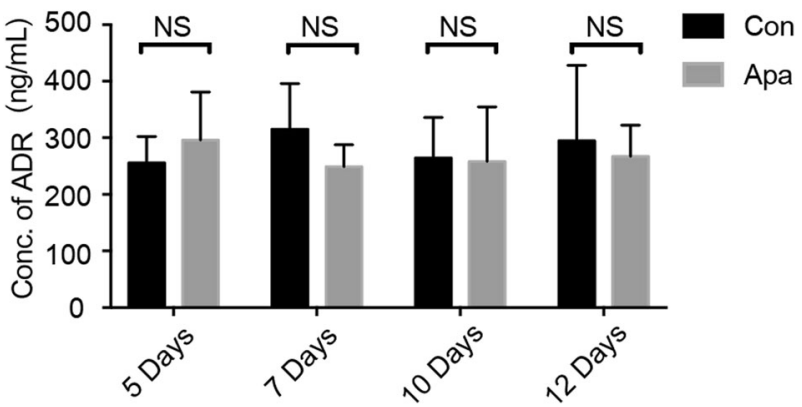

Fig. 5 Effect of Apa on the intratumoral distribution of ADR. a Quantitative measurements of ADR in the tumors by LC-MS/MS $(n=5)$. ${ }^{*} P<0.05$ vs. the Con group. b Quantitative measurements of ADR in the plasma by LC-MS/MS $(n=5)$. NS, not significant certain time window; therefore, it may be crucial to optimize the timing of combined chemotherapy to achieve optimal therapeutic effects.multi-photon imaging and MVD experiments. The inhibition of the VEGF pathway can also decrease the gaps between endothelial cells [26]. As the internalization of adherens junction proteins, such as VE-cadherin, can be activated by VEGF, the inhibition of the VEGF/VEGFR2 pathway may cause endothelial cells to remain in a quiescence state and thus alleviate the leakage of tumor vessels [27] Our SEM and FITC-dextran leakage data demonstrated that Apa improved tumor vessel leakage. Furthermore, the increased pericyte coverage after Apa treatment suggested the improved maturity of the tumor vessels. As more pericytes are recruited around the endothelial cells, the vessel leakage can be further improved, and the subsequent decrease of the interstitial fluid pressure (IFP) could result in reduced obstruction of drug delivery into the tumor [28]. The improvement of the intratumoral delivery of ADR administered in combination with other agents may benefit from the abovementioned changes in the normalization of tumor vessels and micro-environments.

Similar to other reported normalization windows for antiangiogenesis agents, such as bevacizumab, DC 101, and sunitinib $[23,29,30]$, the window for Apa was also limited in time. In the present study, the beginning of the window was 7 days of treatment based on the time of the initiation of tumor vessel structure normalization. It is easy to understand that improved vessel structure allows for the delivery of greater concentrations of chemotherapy drugs into the tumor from the blood vessels. Our results demonstrated that the tumor vessels remained stable and normalized after 12 days of treatment, whereas the ADR distribution and blood perfusion in the tumor decreased to quite low levels that were similar to those observed in the Con group. Notably, blood perfusion was significantly decreased when the normalization window vanished, which might be the major cause 


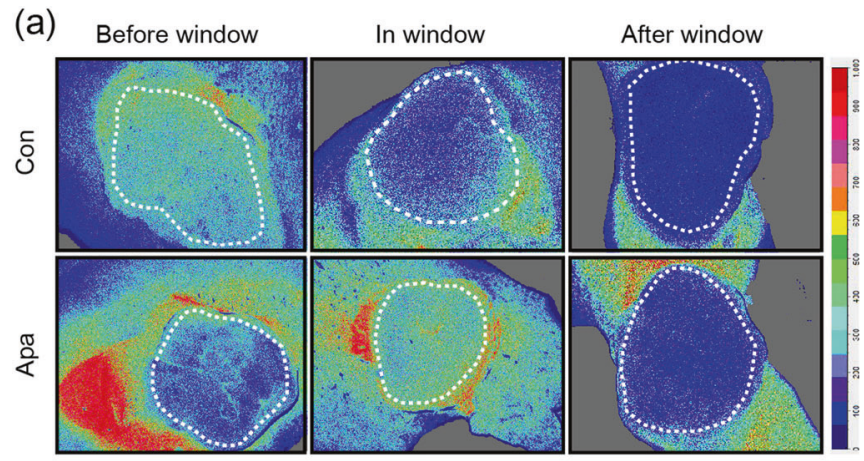

(c) Before window

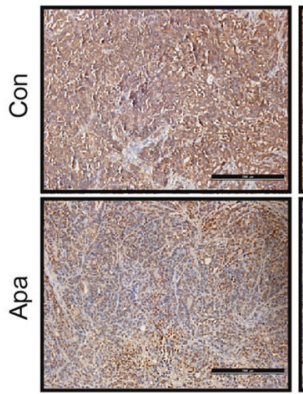

In window

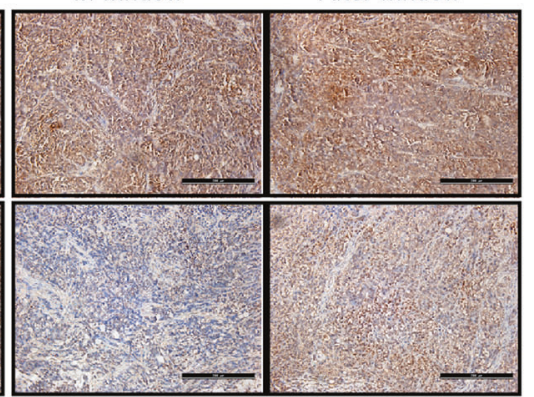

(b)

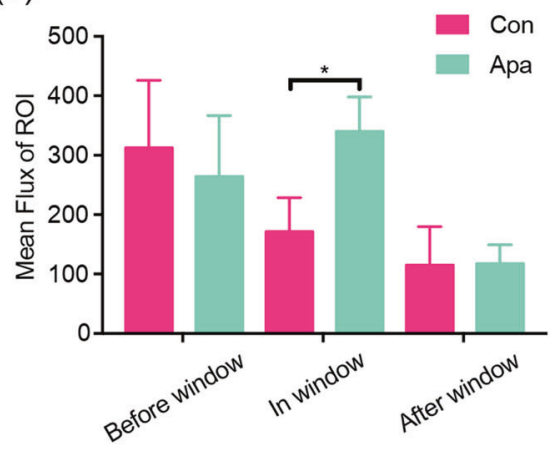

(d)

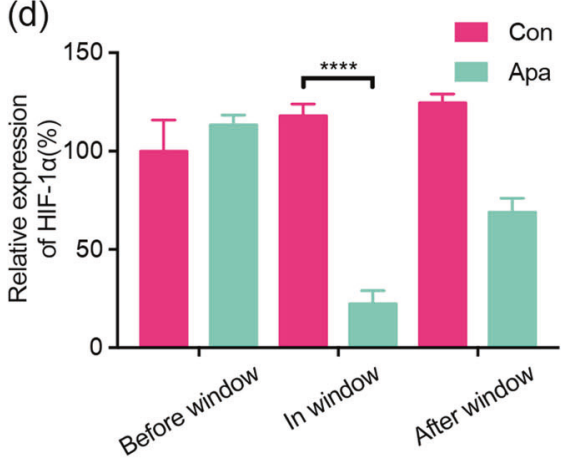

Fig. 6 Blood perfusion and HIF-1 $\alpha$ expression in the tumors. a Blood perfusion in the tumors as monitored by moorFLPI. b Statistical quantification of the blood perfusion in the tumors at different time points $(n=4)$. ${ }^{*} P<0.05$ vs. the Con group. c Immunohistochemical staining for HIF-1 $\alpha$ (scale bar, $200 \mu \mathrm{m})$. d Statistical quantification of the expression of HIF-1 $\alpha$ with ImageJ $(n=4)$. ${ }^{* * * *} P<0.0001$ vs. the Con group

of the reduction in ADR delivery. Alleviated hypoxia in the tumor after the window stage may also help to confirm our assumption. Regarding the reason for the reduced perfusion, it should be attributable to the packing of stromal cells in the tumor. Jain [31] put forward the concept of 'solid stress' caused by stromal cells, which suggests that the compression of the tumor blood vessels is due to solid stress rather than the IFP. A physical force that squeezes the tumor vessels can reduce or even halt the blood flow regardless of whether the vessel structure is intact. Experiments in animal models have demonstrated that targeting collagen and hyaluronan with the angiotensin inhibitor losartan can decompress tumor vessels to increase vascular perfusion [32]. In 2014, a clinical trial targeting solid stress was initiated and involved combining losartan treatment with standard chemotherapy in patients [33]. The mechanisms underlying the initiation and disappearance of the normalization window for Apa need further investigation. Moreover, the discovery of biomarkers of the initiation and disappearance of the normalization window may contribute to guiding the timing of combined drug treatment.

In conclusion, Apa improved the intra-tumor concentration of ADR by normalizing tumor microvessels and micro-environments within a certain time window. These findings provide direct evidence of the tumor vascular normalization effect of Apa and may thus help to establish a schedule for combination therapy in the clinic.

\section{ACKNOWLEDGEMENTS}

This research was financially supported by National Science Foundation of China (81573496, 81773989, 81530098, and 81573494), China "Creation of New Drugs" Key Technology Projects (2015ZX09501001), Jiangsu Province Nature Science Foundation (BK20160076), Postgraduate Research \& Practice Innovation Program of Jiangsu Province (KYLX16_1203), and the Project Program of State Key Laboratory of Natural Medicines, China Pharmaceutical University (SKLNMZZCX201608). We also appreciated the help from Dr. Lei He (Southeast University, China) for her kindly assists on working with multi-photon laser scanning microscope.

\section{AUTHOR CONTRIBUTIONS}

$F Z$ and $G-j W$ designed the research. $K Z, J-w Z$, and FZ performed the research and wrote the paper. J-wZ, Q-yW, W-yL, J-iL, LY, M-mC, S-yN, and Q-yC participated in parts of the research. Competing interests: The authors declare no competing interests.

\section{ADDITIONAL INFORMATION}

Competing interests: The authors declare no competing interests.

\section{REFERENCES}

1. Hanahan D, Weinberg RA. Hallmarks of cancer: the next generation. Cell. 2011;144:646-74.

2. Folkman J. Tumor angiogenesis: therapeutic implications. $\mathrm{N}$ Engl J Med. 1971;285:1182-86.

3. Rao N, Lee YF, Ge R. Novel endogenous angiogenesis inhibitors and their therapeutic potential. Acta Pharmacol Sin. 2015;36:1177-90.

4. Miao Z, Feng J, Ding J. Newly discovered angiogenesis inhibitors and their mechanisms of action. Acta Pharmacol Sin. 2012;33:1103-11.

5. Jain RK. Normalizing tumor vasculature with anti-angiogenic therapy: a new paradigm for combination therapy. Nat Med. 2001;7:987-89.

6. Dickson PV, Hamner JB, Sims TL, Fraga $\mathrm{CH}, \mathrm{Ng} \mathrm{CY}$, Rajasekeran $\mathrm{S}$, et al. Bevacizumab-induced transient remodeling of the vasculature in neuroblastoma xenografts results in improved delivery and efficacy of systemically administered chemotherapy. Clin Cancer Res. 2007;13:3942-50.

7. Tolaney SM, Boucher Y, Duda DG, Martin JD, Seano G, Ancukiewicz M, et al. Role of vascular density and normalization in response to neoadjuvant bevacizumab and chemotherapy in breast cancer patients. Proc Natl Acad Sci USA. 2015;112:14325-30.

8. Jain RK. Antiangiogenesis strategies revisited: from starving tumors to alleviating hypoxia. Cancer Cell. 2014;26:605-22.

9. Goel S, Duda DG, Xu L, Munn LL, Boucher Y, Fukumura D, et al. Normalization of the vasculature for treatment of cancer and other diseases. Physiol Rev. 2011;91:1071-21. 
10. Arjaans M, Schröder CP, Oosting SF, Dafni U, Kleibeuker JE, de Vries EG. VEGF pathway targeting agents, vessel normalization and tumor drug uptake: from bench to bedside. Oncotarget. 2016;7:21247.

11. Franco M, Man S, Chen L, Emmenegger U, Shaked Y, Cheung AM, et al. Targeted anti-vascular endothelial growth factor receptor-2 therapy leads to short-term and long-term impairment of vascular function and increase in tumor hypoxia. Cancer Res. 2006;66:3639-48.

12. Dobosz M, Ntziachristos V, Scheuer W, Strobel S. Multispectral fluorescence ultramicroscopy: three-dimensional visualization and automatic quantification of tumor morphology, drug penetration, and antiangiogenic treatment response. Neoplasia. 2014;16:1W1-13W7.

13. Tian S, Quan H, Xie C, Guo H, Lü F, Xu Y, et al. YN968D1 is a novel and selective inhibitor of vascular endothelial growth factor receptor-2 tyrosine kinase with potent activity in vitro and in vivo. Cancer Sci. 2011;102:1374-80.

14. Li J, Qin S, Xu J, Guo W, Xiong J, Bai Y, et al. Apatinib for chemotherapy-refractory advanced metastatic gastric cancer: results from a randomized, placebo-controlled, parallel-arm, phase II trial. J Clin Oncol. 2013;31:3219-25.

15. Li F, Zhu T, Cao B, Wang J, Liang L. Apatinib enhances antitumour activity of EGFR-TKIs in non-small cell lung cancer with EGFR-TKI resistance. Eur J Cancer. 2017;84:184-92.

16. Song Z, Lin Y, Xia Zhang CF, Lu Y, Gao Y, Dong C. Cyclic RGD peptide-modified liposomal drug delivery system for targeted oral apatinib administration: enhanced cellular uptake and improved therapeutic effects. Int J Nanomed. 2017;12:1941-58.

17. Peng QX, Han YW, Zhang YL, Hu J, Fan J, Fu SZ, et al. Apatinib inhibits VEGFR-2 and angiogenesis in an in vivo murine model of nasopharyngeal carcinoma. Oncotarget. 2017;8:52813.

18. Tong $X$, Wang $F$, Liang $S$, Zhang $X$, He JH, Chen XG, et al. Apatinib (YN968D1) enhances the efficacy of conventional chemotherapeutical drugs in side population cells and $A B C B 1$-overexpressing leukemia cells. Biochem Pharmacol. 2012;83:586-97.

19. Mi $Y$, Liang $Y$, Huang $H$, Zhao HY, Wu CP, Wang F, et al. Apatinib (YN968D1) reverses multidrug resistance by inhibiting the efflux function of multiple ATPbinding cassette transporters. Cancer Res. 2010;70:7981-91.

20. Zhang $M$, Tian $Z$, Sun $Y$. Successful treatment of ovarian cancer with apatinib combined with chemotherapy: a case report. Medicine. 2017;96:e8570.

21. Luo C, Shen J, Ying J, Fang X, Wang X, Fu Z, et al. Case report of a KIT-mutated melanoma patient with an excellent response to apatinib and temozolomide combination therapy. OncoTargets Ther. 2017;10:4553-7.
22. Wilson WR, Hay MP. Targeting hypoxia in cancer therapy. Nat Rev Cancer. 2011;11:393-410.

23. Winkler F, Kozin SV, Tong RT, Chae SS, Booth MF, Garkavtsev I, et al. Kinetics of vascular normalization by VEGFR2 blockade governs brain tumor response to radiation: role of oxygenation, angiopoietin-1, and matrix metalloproteinases. Cancer Cell. 2004;6:553-63.

24. Li N, Zheng D, Wei X, Jin Z, Zhang C, Li K. Effects of recombinant human endostatin and its synergy with cisplatin on circulating endothelial cells and tumor vascular normalization in A549 xenograft murine model. J Cancer Res Clin. 2012;138:1131-44.

25. Vangestel C, Van de Wiele C, Van Damme N, Staelens S, Pauwels P, Reutelingsperger $\mathrm{CP}$, et al. $99 \mathrm{mTC}-(\mathrm{CO}) 3$ His-annexin A5 micro-SPECT demonstrates increased cell death by irinotecan during the vascular normalization window caused by bevacizumab. J Nucl Med. 2011;52:1786-94.

26. Bekes I, Friedl TWP, Köhler T, Möbus V, Janni W, Wöckel A, et al. Does VEGF facilitate local tumor growth and spread into the abdominal cavity by suppressing endothelial cell adhesion, thus increasing vascular peritoneal permeability followed by ascites production in ovarian cancer. Mol Cancer. 2016; 15:13.

27. Gavard J. Endothelial permeability and VE-cadherin: a wacky comradeship. Cell Adhes Migr. 2013;7:465-71.

28. Tolaney SM, Boucher Y, Duda DG, Martin JD, Seano G, Ancukiewicz M, et al. Role of vascular density and normalization in response to neoadjuvant bevacizumab and chemotherapy in breast cancer patients. Proc Natl Acad Sci USA. 2015;112:14325-30.

29. Zhang L, Takara K, Yamakawa D, Kidoya H, Takakura N. Apelin as a marker for monitoring the tumor vessel normalization window during antiangiogenic therapy. Cancer Sci. 2016;107:36-44.

30. Matsumoto S, Batra S, Saito K, Yasui H, Choudhuri R, Gadisetti C, et al. Antiangiogenic agent sunitinib transiently increases tumor oxygenation and suppresses cycling hypoxia. Cancer Res. 2011;71:6350-9.

31. Jain RK. An indirect way to tame cancer. Sci Am. 2014;310:46.

32. Chauhan VP, Boucher Y, Ferrone CR, Roberge S, Martin JD, Stylianopoulos T, et al. Compression of pancreatic tumor blood vessels by hyaluronan is caused by solid stress and not by interstitial fluid pressure. Cancer Cell. 2014;26: 14-5.

33. Chauhan VP, Martin JD, Liu H, Lacorre DA, Jain SR, Kozin SV, et al. Angiotensin inhibition enhances drug delivery and potentiates chemotherapy by decompressing tumour blood vessels. Nat Commun. 2013;4:2516. 